\title{
REPORT
}

\section{Local dispersal can facilitate coexistence in the presence of permanent spatial heterogeneity}

Robin E. Snyder* and Peter Chesson

Section of Evolution and

Ecology, University of

California, Davis, CA 95616, USA

*Correspondence:

E-mail: resnyder@ucdavis.edu

\begin{abstract}
In the presence of permanent spatial heterogeneity, local dispersal, especially short-range dispersal, can facilitate coexistence by concentrating low-density species in the areas where their rates of increase are higher. We present a framework for predicting the effects of local dispersal on coexistence for arbitrary forms of dispersal and arbitrary spatial patterns of environmental variation. Using the lottery model as an example, we find that local dispersal contributes to coexistence by enhancing the effects of environmental variation on scales longer than typical dispersal distances, which can be characterized solely by the variance of the dispersal kernel. Higher moments of the dispersal kernel are not important.
\end{abstract}

\section{Keywords}

Coexistence, dispersal, spatial ecology, spatial heterogeneity.

Ecology Letters (2003) 6: 301-309

\section{INTRODUCTION}

Local dispersal in a spatially heterogeneous environment can cause population levels to build up in favourable areas, which enhances coexistence mechanisms that are present when species differ in their responses to the environment. However, many models employ global dispersal for the sake of tractability and hence underestimate the possibilities for coexistence. Of the studies that consider how local dispersal affects species coexistence, most of them focus on the capacity of local interactions to generate aggregated population distributions in a uniform environment, so that competing species are segregated to some degree (Hassell et al. 1994; Comins \& Hassell 1996; Durrett \& Levin 1997; Kerr et al. 2002; Murrell \& Law 2003). Spatial variation in relative abundance is an important factor in coexistence (Ives 1995); however, we are concerned with how local dispersal can concentrate a low-density species in those areas of a heterogeneous environment that most favour it. The few studies which treat local dispersal in a heterogeneous environment give conflicting results (Comins \& Noble 1985; Bolker 2002). We explain these differences and present a framework capable of dealing with a broad variety of dispersal types and, explicitly spatial environmental variation, to derive some general insights into the effects of local dispersal on species coexistence.

Both long-lasting environmental heterogeneity and species-specific responses to the environment are necessary for local dispersal to enhance the likelihood of coexistence. If the distribution of favourable habitat is different for each species - i.e. there are species-specific environmental responses - then a species which has become temporarily sparse will be able to exploit areas that favour it with relatively little interspecific competition (because the area is not so favourable to its competitors) or intraspecific competition (because it is sparse). In contrast, individuals from a more plentiful species face relatively strong intraspecific competition when in a favourable area. In other words, there is a strong, positive covariance between environmental conditions and competition for the plentiful species but only a weak covariance for the sparse species. Such differences in covariance between environment and competition drive the 'spatial storage effect' (Chesson 2000a) mechanism for coexistence, which can be present even with global dispersal. If the environmental differences are permanent (persistent over multiple generations), then local dispersal helps concentrate the sparse species in the areas that favour it and where it is least limited by interspecific competition. In this way, spatial variation and local dispersal boost the average per capita birth rates of sparse species and facilitate coexistence.

The spatial storage effect and the effects of local population buildup are quantified in Chesson (2000a) based on a generic, discrete-time model. If we define $n_{j x}^{(p r)}(t+1)$ as the expected density of species $j$ offspring produced at location $x$ at time $t+1$, then 
$n_{j x}^{(p r)}(t+1)=\lambda_{j x}(t) n_{j x}(t)$,

where $n_{j x}(t)$ is the expected density of species $j$ at location $x$ at time $t$ and $\lambda_{j x}(t)$ is the finite rate of increase of species $j$ at location $x$ at time $t$. The offspring then disperse, and $n_{j x}(t+1)$ represents the expected density of species $j$ at location $x$ and time $t+1$ after dispersal. Note that in formulating the dynamics in terms of expected density, we are ignoring demographic stochasticity. This should have little effect unless the number of individuals at a site is very low.

We can shift from the local scale to the global scale by taking a spatial average (indicated by an overbar):

$\bar{n}_{j}(t+1)=\overline{\lambda_{j} n_{j}(t)}=\overline{\lambda_{j}} \overline{n_{j}}+\operatorname{Cov}\left(\lambda_{j}, n_{j}\right)=\tilde{\lambda}_{j} \overline{n_{j}}(t)$,

where

$\tilde{\lambda}_{j}=\bar{\lambda}_{j}+\operatorname{Cov}\left(\lambda_{j}, v_{j}\right)$

$v_{j x}=\frac{n_{j x}}{\bar{n}_{j}}$.

That is, the global dynamics are like the local production dynamics but with an effective finite rate of increase $\tilde{\lambda}_{j}$ equal to the spatial average of the local rate of increase $\lambda_{j x}$ plus the covariance between local growth and the relative population density $v_{j x}$. The relative population density is simply the ratio of the local density to the average density over all locations. The growth-density covariance is taken over space and accounts for the fact that population levels may be higher in some locations than in others, and the rate of increase in these more populous spots will contribute more to the overall rate of increase of the population. We assume that species coexist if each species can invade when the other species are at the equilibrium spatial distributions that they would attain in the absence of the invader. This means that each species has a finite rate of increase greater than 1 when it is reduced to low density [the standard invasibility criterion (Gotelli 1995; Chesson 2000a)]. Thus, the condition for coexistence is that $\tilde{\lambda}>1$ for each species as an invader. The contribution of the growth-density covariance to $\tilde{\lambda}$ demonstrates the importance of local dispersal and an explicitly spatial representation. Local dispersal can cause population levels to build up in favourable areas, which makes the growth-density covariance, $\operatorname{Cov}\left(\lambda_{j}, v_{j}\right)$, positive. This covariance increases the global finite rate of increase, $\tilde{\lambda}_{j}$, and thereby promotes coexistence.

In analogy with ANOVA techniques, the finite rate of increase $\tilde{\lambda}_{j}$ can be further partitioned into the primary effects of the environment, competition, and the interaction between environment and competition (Chesson 2000a). Additional manipulations then allow one to re-express $\tilde{\lambda}_{j}$ as the sum of effects that do not depend on spatial variation and the effects of three spatial mechanisms: 'relative nonlinearity' in local rates of increase, the spatial storage effect and the growth-density covariance $\left[\operatorname{Cov}\left(\lambda_{j}, v_{j}\right)\right]$, which enhances the spatial storage effect (Chesson 2000a). Spatially independent coexistence mechanisms, relative nonlinearity and the spatial storage effect contribute to $\bar{\lambda}_{j}$, and can be present with global dispersal and local dispersal. The effects of local dispersal - the effects of an explicitly spatial representation - are contained entirely within $\operatorname{Cov}\left(\lambda_{j}, v_{j}\right)$ for the model we use here, although in general, local dispersal can contribute to components of $\bar{\lambda}$ as well.

In this paper, we use the framework of Chesson (2000a) to explore the effects of local dispersal on species coexistence. We present an expression for the growthdensity covariance for arbitrary probability distributions of dispersal distance (dispersal kernels) and arbitrary patterns of environmental variation. Using the lottery model (defined below) as an example, we demonstrate how the parameter ranges where coexistence is possible increase when one considers local instead of global dispersal, and we highlight the effects of different forms of dispersal and different patterns of environmental variation. We find in particular that the contribution of local dispersal to coexistence depends only on environmental variation at scales longer than typical dispersal distances and that shortrange dispersal facilitates coexistence most effectively. Furthermore, the variance of the dispersal kernel is sufficient to characterize dispersal distances - one need not measure higher moments.

The rest of this paper is organized as follows. In 'The lottery model', we define our example, the lottery model. We present a general expression for the growth-density covariance in 'An expression for the growth-density covariance', and discuss the implications of this expression in 'Implications'. 'Synthesis of spatial mechanisms' is devoted to a review of the previous work on coexistence in a heterogeneous environment in the light of this work. We conclude in 'Discussion' with a discussion of our work and an outline of future directions.

\section{THE LOTTERY MODEL}

We demonstrate our results with the lottery model (Chesson \& Warner 1981; Comins \& Noble 1985; Chesson 2000a; Muko \& Iwasa 2000), a model commonly used to understand the effects of environmental variation on competitive interactions. Here, we use a spatial version of the model with dispersing juveniles that compete for space to become sessile, semelparous adults. The lottery model is one of the simplest models with which one can demonstrate the effects of a varying environment and so has achieved the status of a standard example, much like the Lotka-Volterra 
model or the contact process. The lottery model is most frequently used to represent marine organisms such as sessile invertebrates or coral reef fish. In this paper, we assume that adults die after reproducing, and so here, the model is perhaps best thought of in terms of annual plants. In the case of annual plants, 'juveniles' are seeds.

For the sake of simplicity, we assume that the environment is one-dimensional and can be represented as a series of patches, each of which can support a single individual. The ability of juveniles of a given species to capture space is a function of the environment type, which is assumed to vary in space but not time. The space-capturing ability of species $j$ at location $x$ is denoted by $E_{j x}$. For annual plants, $E_{j x}$ could represent the product of germination probability and early seedling survival probability.

Let $n_{j x}(t)$ be the expected density of seeds of species $j$ competing for space at location $x$ at time $t$. We imagine that competition for a site is like a lottery, with the winner chosen randomly from the seeds competing at that site and the chances of a species seizing the site proportional to the number of seeds it has in the competition. This is not a fair lottery, however. For any given site, seeds from some species will be more likely to win than those of other species, with their advantage determined by $E_{j x}$. Mathematically, the expected number of species $j$ individuals that establish themselves at $x$ and become adults is

$\frac{E_{j x} n_{j x}}{\sum_{k} E_{k x} n_{k x}}$,

where the sum in the denominator runs over all species. The per adult fecundity of species $j$ is $F_{j}$, so that the number of species $j$ seeds produced at $x$ that compete at time $t+1$ is

$n_{j x}^{(p r)}(t+1)=\frac{F_{j} E_{j x} n_{j x}}{\sum_{k} E_{k x} n_{k x}}$.

The mathematical framework introduced in Chesson (2000a) assumes that local reproduction takes the form $n_{j x}^{(p r)}(t+1)=\lambda_{j x} n_{j x}(t)$, and so

$\lambda_{j x}=\frac{F_{j} E_{j x}}{\sum_{k} E_{k x} n_{k x}}$.

Equation 6 takes the initial number of seeds of each species, $n_{j x}$, as given and does not include seed dispersal. After reproduction, seeds disperse to the left and right, with the probability of dispersing from $x$ to $x+z$ given by the dispersal kernel, $k(z)$. Thus, combining dispersal with reproduction, we find

$n_{j x}(t+1)=\sum_{y} k(x-y) \lambda_{j y} n_{j y}(t)$.

Equation 8 states that the number of individuals landing at location $x$ at time $t+1$ is equal to the number of individuals produced at location $y$ at time $t\left(\lambda_{j j} n_{j y}(t)\right)$ times the probability of dispersing from $y$ to $x(k(x-y))$, summed over all $y$. We want to find $\operatorname{Cov}\left(\lambda_{j x}, v_{j x}\right)$, so we need eqn 8 not in terms of population density, $n_{j x}$, but in terms of relative population density, $v_{j x}(t)=n_{j x}(t) / \bar{n}_{j}(t)$. A small amount of algebra yields

$v_{j x}(t+1)=\sum_{y} k(x-y) \frac{\lambda_{j y}}{\tilde{\lambda}_{j}} v_{j y}(t)$.

Note that eqn 9 is valid for all models of the form $n_{j x}^{(p r)}(t+1)=\lambda_{j x} n_{j x}(t)$. Here we use the lottery model by taking eqn 7 as our definition of $\lambda_{j j}$.

In the next section, we restrict our attention to the case of two species, and it will be useful to derive a few two-species results here. First, consider the dynamics of the resident in the absence of the invader:

$n_{r x}(t+1)=\frac{F_{r} E_{r x} n_{r x}}{E_{r x} n_{r x}}=F_{r}$.

We see that in a single step, the resident population achieves a spatially uniform equilibrium distribution, $n_{r x}^{*}=F_{r}$. Then, given a spatially uniform production of offspring, dispersal preserves the uniformity of their distribution in space, although individuals do move.

We can now find the invader's local rate of increase:

$\lambda_{i x}=\frac{F_{i} E_{i x}}{E_{r x} n_{r x}^{*}+E_{i x} n_{i x}}$,

where subscripts $i$ and $r$ denote invader and resident quantities and $n_{r x}^{*}$ is the equilibrium density of the resident in the absence of the invader. As the invader density is by definition very low, we can set $n_{i x}=0$ in the denominator of eqn 11 , and we know from eqn 10 that $n_{r x}^{*}=F_{r}$. Thus,

$\lambda_{i x}=\frac{F_{i} E_{i x}}{F_{r} E_{r x}}$.

\section{AN EXPRESSION FOR THE GROWTH-DENSITY COVARIANCE}

Environmental variation usually occurs on many spatial scales simultaneously. It would be useful if we could analyse the contribution of each spatial scale to the growth-density covariance separately and sum the contributions to obtain the net effect. Fortunately, Fourier transforms are ideally suited to this task. Fourier transforms decompose functions into a sum of sine waves at different frequencies, and one can loosely think of the Fourier transform of a function, evaluated at spatial frequency $\omega$, as specifying how much of that function can be represented by a sine wave at frequency $\omega$. (Recall that wave length equals $2 \pi / \omega$.) Using Fourier transforms, we can separate the environmental variation into a series of sine waves and analyse the contributions to coexistence of each spatial scale separately. Thanks to the 
additional properties of Fourier transforms, the effects of variation at these different spatial scales are additive.

We measure the environmental variation with the perturbation $\zeta_{i x}=\left(\lambda_{i x} / \bar{\lambda}_{i}\right)-1$, which indicates how the local invader rate of increase differs from its spatial average. Specializing on the case of a lottery model with two species, we use eqn 12 to find $\zeta_{i x}=\left(\lambda_{i x} / \bar{\lambda}_{i}\right)-1=\left[\left(1 / \bar{\lambda}_{i}\right)\left(F_{i} / F_{r}\right)\right.$ $\left.\left(E_{i x} / E_{r x}\right)\right]-1$. Thus, $\zeta_{i x}$ is a measure of how much the ratio of the invader and resident environmental responses varies from spatial uniformity. Our expression for the growth-density covariance will use the spectral density of $\zeta_{i}$, $S_{\zeta}(\omega)$, which is proportional to the square of the Fourier transform of $\zeta_{i}$. (Fourier transforms and spectral densities are defined precisely in Appendix.)

We can now present our primary result:

$\operatorname{Cov}\left(\lambda_{i}, v_{i}\right)=\bar{\lambda}_{i} \frac{1}{2 \pi} \int_{-\pi}^{\pi} \frac{\tilde{k}(\omega) S_{\zeta}(\omega)}{1-\tilde{k}(\omega)} \mathrm{d} \omega$,

where $\tilde{k}(\omega)$ is the Fourier transform of the dispersal kernel and $\operatorname{Cov}\left(\lambda_{i}, v_{i}\right)$ is the standard covariance over space of the two quantities $\lambda_{i}$ and $v_{i}$, i.e. $\left(\lambda_{i}(x)-\bar{\lambda}_{i}\right)\left(v_{i}(x)-\bar{v}_{i}\right)$. Equation 13 states that the growth-density covariance is proportional to $[\tilde{k}(\omega) /(1-\tilde{k}(\omega))]$, which contains information about how much dispersal occurs at frequency $\omega$, summed over frequency, with $S_{\zeta}(\omega)$ weighting each term by how much environmental variation is present at that frequency.

It is the ratio of the invader and resident environments and not their separate values which are important for the lottery model. It therefore makes sense to measure the environmental responses on a logarithmic scale, so that the species are treated symmetrically $\left[\ln \left(E_{1} / E_{2}\right)=-\ln \left(E_{2} / E_{1}\right)\right]$. Let $\ln \left(F_{j} E_{j}\right)=\mu_{j}+\epsilon_{j x}, j=i, r$, where $\mu_{j}$ is constant, and $\epsilon_{j x}$, representing deviations away from $\mu_{j}$, has spatial mean zero. Then $\tilde{\lambda}_{i}$, the invader's global scale finite rate of increase, is

$\tilde{\lambda}_{i}=\left(\mu_{i}-\mu_{r}\right)+\frac{1}{2} \operatorname{Var}\left(\epsilon_{i}-\epsilon_{r}\right)+\operatorname{Cov}\left(\lambda_{i}, v_{i}\right)$.

In the absence of spatial variation, $\tilde{\lambda}_{i}$ would equal the fitness difference, $\mu_{i}-\mu_{r}$ A spatially varying environment adds to $\tilde{\lambda}_{i}$ via $\frac{1}{2} \operatorname{Var}\left(\epsilon_{i}-\epsilon_{r}\right)$ (the storage effect) and $\operatorname{Cov}\left(\lambda_{i}, v_{i}\right)$ (the growth-density covariance). [Relative nonlinearity, another component of $\bar{\lambda}$ defined in (Chesson 2000a), is zero for the lottery model.] The storage effect term and the growth-density covariance are stabilizing terms that, if strong enough, overcome fitness differences and permit coexistence as discussed in Chesson (2000b).

As an example, consider two species, 1 and 2 , whose spacecapturing abilities have the ratio $\ln \left(E_{1 x} / E_{2 x}\right)=b \sin \left(\omega_{e} x\right)$. The choice of a sinusoidally varying environment is decidedly artificial. However, as noted previously, the contributions to coexistence from multiple spatial scales are additive, and so for simplicity, we consider environmental variation at a single

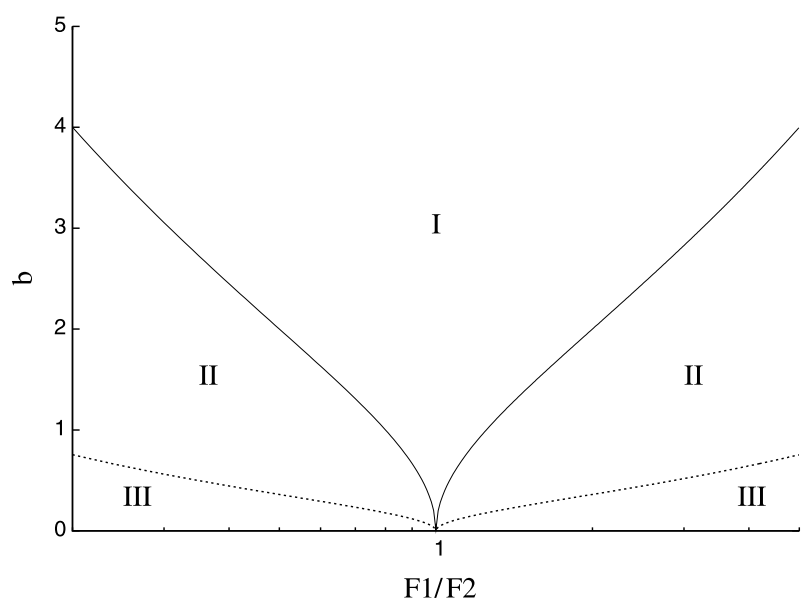

Figure 1 Coexistence region for environmental sensitivity $(b)$ vs. the logarithm of the adult fecundity ratio $\left(F_{1} / F_{2}\right)$. The environment varies according to $\ln \left(E_{1 x} / E_{2 x}\right)=b \sin \left(\omega_{\epsilon} x\right)$. The frequency of environmental variation, $\omega_{e}$, is 0.1 , and is measured in radians. In region $\mathrm{I}$, coexistence is possible even if dispersal is global, while in region II, coexistence is only possible if there is limited dispersal. Coexistence is not possible in region III. The dispersal kernel for this figure had a variance of 13.2. (Only the variance of the dispersal kernel matters. See 'Implication' for a discussion of this.)

frequency. Figure 1 shows how strongly the environment must vary $(b)$ for the species to coexist given a ratio of adult fecundities $\left(F_{1} / F_{2}\right)$. Recall that for a species to coexist, $\tilde{\lambda}$ must be greater than 1 for both species, where $\tilde{\lambda}=\bar{\lambda}+\operatorname{Cov}\left(\lambda_{i}, v_{i}\right)$. In region I, both species can coexist even if dispersal is global. Here, $\bar{\lambda}_{i}>1$ for both species as invaders; the spatial storage effect is strong enough to overcome the fitness disadvantage of the inferior competitor. In region II, both species can coexist if there is limited dispersal. (This figure was generated using a particular choice of dispersal kernel, but, as discussed in 'Implications', any kernel with the same variance would result in the same boundary.) Here, $\bar{\lambda}_{i}<1$, but $\operatorname{Cov}\left(\lambda_{i}, v_{i}\right)$ lifts $\tilde{\lambda}$ above 1 . This is the extra coexistence region provided by $\operatorname{Cov}\left(\lambda_{i}, v_{i}\right)$. In region III, coexistence is not possible.

\section{IMPLICATIONS}

The way information about dispersal enters our expression for growth-density covariance has important consequences. Our knowledge of dispersal is contained in the Fourier transform of the dispersal kernel, $\tilde{k}(\omega)$. For all kernels, $\tilde{k}(0)=1$, as the Fourier transform of a function evaluated at zero frequency is simply the integral of the function from $-\infty$ to $\infty$, and, being probability distributions, all kernels integrate to 1 . The transform $\tilde{k}(\omega)$ is close to 1 for $\omega$ close to zero [for $\omega$ small, $\tilde{k}(\omega) \approx 1-\frac{1}{2} \operatorname{Var}(k) \omega^{2}$ ], and so 
$\tilde{k}(\omega) /(1-\tilde{k}(\omega))$ is large when $\omega$ is small ${ }^{1}$. These large values dominate the integral in eqn 13 . This has two important implications.

First, as the integral in eqn 13 is dominated by low frequencies, we can replace $\tilde{k}(\omega)$ by the first two terms of its Taylor expansion about $\omega=0: \tilde{k}(\omega) \approx 1-\frac{1}{2} \operatorname{Var}(k) \omega^{2}$. Thus, eqn 13 becomes

$\operatorname{Cov}\left(\lambda_{i}, v_{i}\right)=\bar{\lambda}_{i} \frac{1}{2 \pi} \int_{-\pi}^{\pi} \frac{S_{\zeta}(\omega)\left(2-\operatorname{Var}(k) \omega^{2}\right)}{\operatorname{Var}(k) \omega^{2}} \mathrm{~d} \omega$.

Unless all the environmental variation is at frequencies that are high relative to $1 / \sqrt{\operatorname{Var}(k)}$, this approximation is highly accurate: none of the figures in this paper would change by more than a penwidth were we to use this approximation. This means that we can ignore higher order moments, which depend increasingly on the hardto-measure tails of the dispersal kernel, and rely simply on the variance, which is much easier to estimate. This is in contrast to invasion studies, in which the invasion speed is determined largely by the tails of the invader's dispersal kernel (Mollison 1991; Kot et al. 1996). Figure 2 shows how the coexistence region depends on $\sqrt{\operatorname{Var}(k)}$.

Second, only environmental variation at wavelengths longer than typical dispersal distances contributes much to $\operatorname{Cov}\left(\boldsymbol{\lambda}_{i}, v_{i}\right)$. Mathematically, this is easy. The factor $\left[\left(2-\operatorname{Var}(k) \omega^{2}\right) /\left(\operatorname{Var}(k) \omega^{2}\right)\right]$ is large for $1 / \omega \gg \sqrt{\operatorname{Var}(k)}$,

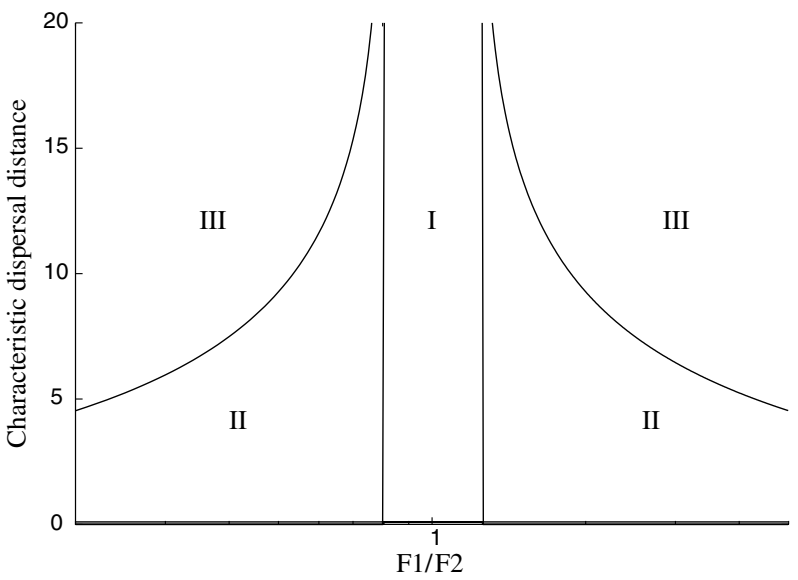

Figure 2 Coexistence region for characteristic dispersal distance $[\sqrt{\operatorname{Var}(k)}]$ vs. the logarithm of the adult fecundity ratio $\left(F_{1} / F_{2}\right)$. The environment varies according to $\ln \left(E_{1 x} / E_{2 x}\right)=b \sin \left(\omega_{e} x\right)$. The frequency of environmental variation, $\omega_{e}$, is 0.1 , and is measured in radians, while $b=1$. In region $\mathrm{I}$, coexistence is possible even if dispersal is global, while in region II, coexistence is only possible if there is limited dispersal. Coexistence is not possible in region III.

${ }^{1}(\tilde{k}(\omega) /(1-\tilde{k}(\omega))$ is infinite when $\omega=0$. However, for nonpathological examples, $S_{\zeta}(\omega)=0$ when $\omega=0$, and so the potential pole is suppressed.

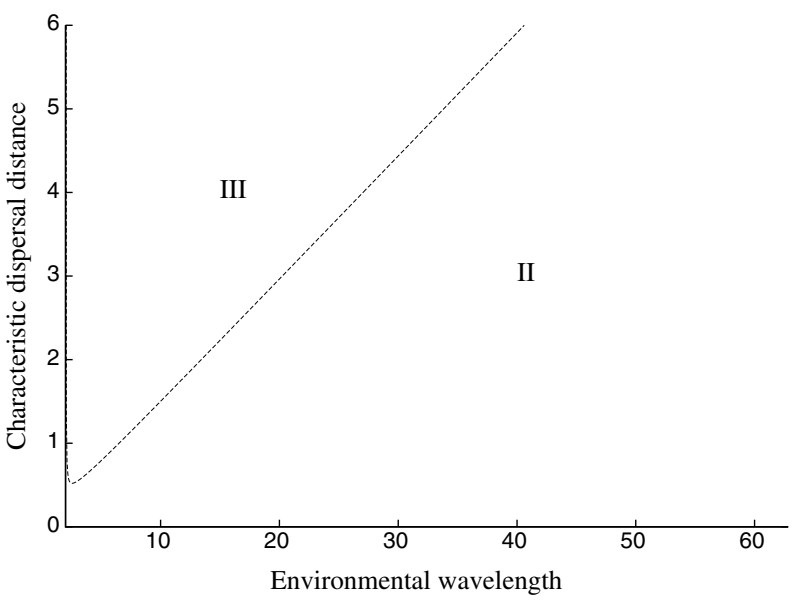

Figure 3 Coexistence region for characteristic dispersal distance $[\sqrt{\operatorname{Var}(k)}]$ vs. the wavelength of the environmental variation. The environment varies according to $\ln \left(E_{1 x} / E_{2 x}\right)=b \sin \left(\omega_{e} x\right)$. The ratio of adult fecundities $\left(F_{1} / F_{2}\right)$ is 2 , while $b=1$. Coexistence is not possible with global dispersal, but is possible with limited dispersal in region II. Coexistence is not possible in region III.

and the standard deviation of the dispersal kernel, $\sqrt{\operatorname{Var}(k)}$, can be thought of as a characteristic dispersal distance. Wavelength equals $2 \pi / \omega$, and so this inequality means that the factor $\left[\left(2-\operatorname{Var}(k) \omega^{2}\right) /(\operatorname{Var}(k))\right]$ gives a lot of weight to wavelengths much larger than the characteristic dispersal distance. This finding stresses the importance of short-range dispersal, because it means that smaller values of $\operatorname{Var}(k)$ give larger ranges of frequencies at which environmental variation contributes significantly to the growth-density covariance and give stronger contributions at those frequencies. This finding also makes sense biologically. If favourable areas are large relative to typical dispersal distances, then offspring of adults in favourable areas are likely to remain in those areas, which encourages population buildup there and enhances the spatial storage effect. Figure 3 shows coexistence regions as a function of the characteristic dispersal distance, $\sqrt{\operatorname{Var}(k)}$, and the wavelength of the environmental variation.

\section{SYNTHESIS OF SPATIAL MECHANISMS}

Our work extends the findings of studies that consider species coexistence in a lottery model with permanent spatial heterogeneity but confine themselves to global dispersal for the sake of tractability (Chesson 1985; Muko \& Iwasa 2000). Not only have we shown how to incorporate local dispersal and identified the importance of population buildup in favourable areas, but, by thinking in terms of the storage effect, we can also give intuitive explanations for results such as the Muko and Iwasa 
finding that although spatial variation in mortality enables coexistence, variation in fecundity does not (Muko \& Iwasa 2000). As dispersal is global, juveniles competing in a given patch come from all over the system. As there is no connection between fecundity in that patch and the competition experienced by juveniles that have landed there, plentiful species do not pay the price of high intraspecific competition in favourable areas. In the terminology of the storage effect, there is no covariance between the environment experienced and the competition experienced. Note, however, that if dispersal were local, so that competing juveniles were more likely to have come from the patch in question than from any other patch, then population levels would build up in high fecundity sites, and the resulting increase in competition would limit output from those sites. Thus, spatially varying fecundity could enable coexistence in the presence of local dispersal. Alternatively, variation in mortality can introduce covariance between environment and competition in the presence of either global or local dispersal. Where the environment favours adult persistence, there will be few vacancies for juveniles to occupy, and competition will be strong. The sedentary nature of the adults acts much like local retention of juveniles.

This work also extends studies which allow for some fraction of the offspring to be retained locally while the others disperse globally, such as in Chesson (2000a) and Comins \& Noble (1985). Such stark representations of local retention offer a very clearcut, if unrealistic, demonstration of the importance of local buildup. It is shown in Chesson (2000a)) that this kind of local retention leads to a positive $\operatorname{Cov}\left(\lambda_{i}, v_{i}\right)$.

One way researchers have incorporated explicit space without adding too much complexity is by limiting their arena to two patches. Our findings parallel those of twopatch studies of coexistence, such as Pacala \& Roughgarden (1982) and Amarasekare \& Nisbet (2001). Amarasekare and Nisbet present a model with two patches of different quality, Lotka-Volterra competitive dynamics, and migration between patches at constant rates (Amarasekare \& Nisbet 2001). They find that for two of their three situations, dispersal rates must be below critical thresholds for species to coexist. Likewise, Pacala and Roughgarden, working with diffusive movement in a two-patch competition model, found that dispersal of the resident increases competition in the habitat with the lower carrying capacity (Pacala \& Roughgarden 1982). If an invader disperses into this area too frequently, then it is overwhelmed by the competition, and coexistence is not possible. We can restate the results of both of these studies by saying that the invader population must be partially retained in the areas which favour it, i.e. the growth-density covariance must be sufficiently positive.
The benefit of our approach over two-patch models is that we can accommodate arbitrary forms of environmental variation and arbitrary forms of dispersal. This helps to ensure the generality of our results as well as yielding insights that are only possible in this framework, such as the importance of kernel variance. One study that follows this more general approach is that of Bolker and Pacala, who investigate coexistence mechanisms in a homogeneous environment (Bolker \& Pacala 1999). Although it is not identified explicitly, $\operatorname{Cov}\left(\lambda_{i}, v_{i}\right)$ plays an important role in their coexistence criteria as well. If we return to the discrete time approximation used in their derivation, we can say that $\bar{n}_{I}(t+d t) \approx \tilde{\lambda}_{I} \bar{n}_{I}(t)$, where

$\tilde{\lambda}_{I}=1+r_{I}\left\{1-\frac{\bar{n}_{I}+\left(\bar{c}_{I I} / \bar{n}_{I}\right)}{K_{I}}-\frac{\beta_{I R}\left[\bar{n}_{R}+\left(\bar{c}_{I R} / \bar{n}_{I}\right)\right]}{K_{R}}\right\} \mathrm{d} t$,

$\bar{n}_{I}$ and $\bar{n}_{R}$ are spatial averages of the invader and resident populations, and $\bar{c}_{I I}$ and $\bar{c}_{I R}$ are spatial covariances averaged over a convolution of the dispersal and competition kernels. After some work, we can identify $\bar{\lambda}_{I}$ as $1+r_{I}\left\{1-\bar{n}_{I} / K_{I}-\right.$ $\left.\beta_{I R} \bar{n}_{R} / K_{R}\right\} \mathrm{d} t$ and $\operatorname{Cov}\left(\lambda_{i}, v_{i}\right)$ as $r_{I}\left[\left(-\bar{c}_{I I} / K_{I} \bar{n}_{I}\right)-\right.$ $\left.\left(\beta_{I R} \bar{c}_{I R} / K_{R} \bar{n}_{I}\right)\right] \mathrm{d} t$. Note that without the $\operatorname{Cov}\left(\lambda_{i}, v_{i}\right)$ term, this would reduce to a Lotka-Volterra competition model. For both Bolker and Pacala's model and ours, the effects of an explicitly spatial representation are contained in $\operatorname{Cov}\left(\lambda_{i}, v_{i}\right)$, and for both studies, $\operatorname{Cov}\left(\lambda_{i}, v_{i}\right)$ features prominently in the coexistence criteria.

Bolker has also discussed heterogeneous environments and has noted how population buildup in favourable areas fortifies species persistence, although he has not addressed species coexistence in this context (Bolker 2002). In particular, he notes that the greater the spatial variability in the environment, the more advantageous short-range dispersal is, as it tends to keep offspring in favourable areas.

As our environmental variation is fixed in time, 'fugitive' strategies, in which an inferior competitor can coexist with a superior competitor by being swifter to discover and colonize newly available habitat (Levins \& Culver 1971; Hastings 1980; Tilman 1994), are not applicable. If there were temporal and spatial variation, we would expect the emphasis on local retention of offspring to change. Comins and Noble consider the lottery model with spatiotemporal variation in the environment (Comins \& Noble 1985). They obtain analytic results for global dispersal and use simulations to investigate nearest-neighbour dispersal. In contrast with our results, they find that dispersal distances must be much larger than the scale of spatial variation in the environment if species are to coexist. The difference arises because they consider pure spatiotemporal variation, in which the environment at each 
location changes over time independently of the environment at other locations. If favourable areas persist, then the best strategy of an organism is to settle offspring nearby, in the hope that they will land in a similarly favourable spot. However, if good patches are ephemeral, then the best strategy is to send offspring far and wide in the hope that a few of them will find a new good patch.

\section{DISCUSSION}

In this paper, we have derived an expression for the covariance between relative population density and local growth and demonstrated how this increases the parameter space over which coexistence is possible. The covariance expression is dominated by the low frequency terms. This has the consequence that local dispersal contributes to coexistence by enhancing the effects of environmental variation on scales longer than typical dispersal distances. Short-range dispersal facilitates coexistence most effectively. Restating this result somewhat differently, the scales of environmental variation that contribute significantly to the growth-density covariance depend on the scale of dispersal. Even if two species experience an identical pattern of environmental variation, the species with shorter-range dispersal will have a larger growth-density covariance because it is sensitive to environmental variation at a greater range of spatial scales and because the contributions within that range will be larger. The domination of the low frequency terms also means that dispersal distances can be characterized solely by the variance of the dispersal kernel higher moments can be ignored. Biologically, this reflects the fact that rare, long-distance dispersal does not appreciably affect the degree of population buildup in favourable locations.

We have seen that short-range dispersal can provide a significant advantage when environmental variation is effectively permanent. If favourable areas are transient (e.g. carrion, puddles), then it is advantageous for at least some offspring to disperse long distances so that they can colonize newly favourable areas before the natal site becomes unfavourable. Such a situation might select for a leptokurtic dispersal kernel, with most offspring staying close to home and a few dispersing broadly. The more rapidly the environment changes, the more important longdistance dispersal will be, so that there may be an ideal balance between short and long-range dispersal for a given turnover rate (Levin et al. 1984).

The view of coexistence presented in this paper is a niche differentiation perspective. Within the context of the lottery model, the niche is Grubb's regeneration niche, and $E_{j}$ represents environmental features favouring the establishment of species $j$ (Grubb 1977). We assume that the environment varies in space and that the different species have different resource needs and tolerances, so that each perceives a different spatial pattern of environmental quality. This variation is a semi-permanent feature of the environment, such as soil type, aspect or exposure, and so we find that the likelihood of coexistence is most enhanced when typical dispersal distances are short with respect to typical lengthscales of environmental variation, so that the offspring of individuals in favourable areas tend to remain in those areas, and populations of each species accumulate in the areas that favour them. Additional mechanisms for coexistence operate in spatially varying environments, such as relative nonlinearity or the spatial storage effect without the enhancement of local population buildup [see Chesson (2000a)], but these do not depend on dispersal distance and are beyond the scope of this paper.

Our expression for the growth-density covariance (eqns 34 and 13) holds not just for the lottery model but for any model that can be written in the form $n_{j x}(t+1)=$ $\sum_{y} k(x-y) \lambda_{j y} n_{j y}(t)$. However, the interpretation of the covariance expression is more complicated if the resident's equilibrium distribution in the absence of the invader $\left(n_{r x}^{*}\right)$ is not spatially uniform. In that case, $S_{\zeta}(\omega)$ will be a function not only of the environmental variation, but also of $n_{r x}^{*}$. While it will still be true that it is the low frequency components of $S_{\zeta}(\omega)$ which matter, we will no longer be able to interpret this simply as long wavelength environmental variation. Similarly, $\bar{\lambda}_{i}$ will depend on $n_{r x}^{*}$, which will make it more difficult to compute. On the other hand, we should still be able to replace the Fourier transform of the dispersal kernel, $\tilde{k}(\omega)$, with $1-\frac{1}{2} \operatorname{Var}(k)$. In the context of the lottery model, $n_{r x}^{*}$ will not be spatially uniform if adult fecundity varies in space, if the number of the individuals able to occupy a site (here set to 1) varies in space, or if juveniles compete not only within a site but also to some degree with juveniles at neighboring sites. These issues are the subjects of future studies.

\section{ACKNOWLEDGMENTS}

This work was supported by NSF grant number NSF DEB99-81926.

\section{REFERENCES}

Amarasekare, P. \& Nisbet, R.M. (2001). Spatial heterogeneity, source-sink dynamics, and the local coexistence of competing species. Am. Nat., 158, 572-584.

Bolker, B. (2002). Combining endogenous and exogenous variability in analytical population models. Theor. Pop. Biol., in press.

Bolker, B.M. \& Pacala, S.W. (1999). Spatial moment equations for plant competition: understanding spatial strategies and the advantages of short dispersal. Am. Nat., 153, 575-602. 
Chesson, P.L. (1985). Coexistence of competitors in spatially and temporally varying environments: a look at the combined effects of different sorts of variability. Theor. Pop. Biol., 28, 263-287.

Chesson, P. (2000a). General theory of competitive coexistence in spatially-varying environments. Theor. Pop. Biol., 58, 211-237.

Chesson, P. (2000b). Mechanisms of maintenance of species diversity. Ann. Rev. Ecol. Syst., 343-366.

Chesson, P.L. \& Warner, R. (1981). Environmental variability promotes coexistence in lottery competitive systems. Am. Nat., 117, 923-943.

Comins, H. \& Hassell, M. (1996). Persistence of multispecies hostparasitoid interactions in spatially distributed models with local dispersal. J. Theor. Biol., 183, 19-28.

Comins, H. \& Noble, I. (1985). Dispersal, variability, and transient niches: species coexistence in a uniformly variable environment. Am. Nat., 126, 706-723.

Durrett, R. \& Levin, S. (1997). Allelopathy in spatially distributed populations. J. Theor. Biol., 185, 165-171.

Gotelli, N.J. (1995). A Primer of Ecology. Sinauer Associates, Sunderland, MA

Grubb, P. (1977). The maintenance of species richness in plant communities: the importance of the regeneration niche. Biol. Rev., 52, 107-145.

Hassell, M.P., Comins, H.N. \& May, R.M. (1994). Species coexistence and self-organizing spatial dynamics. Nature, 370, 290292.

Hastings, A. (1980). Disturbance, coexistence, history, and competition for space. Theor. Pop. Biol., 18, 363-373.

Ives, A.R. (1995). Measuring competition in a spatially heterogeneous environment. American Naturalist, 146, 911-936.

Kerr, B., Riley, M.A., Feldman, M.W. \& Bohannan, B.J. (2002). Local dispersal promotes biodiversity in a real-life game of rockpaper-scissors. Nature, 418, 171-174.

Kot, M., Lewis, M. \& van den Driessche, P. (1996). Dispersal data and the spread of invading organisms. Ecology (Washington, DC), 77, 2027-2042.

Levin, S.A., Cohen, D. \& Hastings, A. (1984). Dispersal strategies in patchy environments. Theor. Pop. Biol., 26, 165-191.

Levins \& Culver (1971). Regional coexistence of species and competition between rare species. Proc. Nat. Acad. Sci. USA, 6, 1246-1248.

Mollison, D. (1991). Dependence of epidemic and population velocities on basic parameters. Math. Biosci., 107, 255-288.

Muko, S. \& Iwasa, Y. (2000). Species coexistence by permanent spatial heterogeneity in a lottery model. Theor. Pop. Biol., 57, 273284.

Murrell, D.J. \& Law, R. (2003). Heteromyopia and the spatial coexistence of similar competitors. Ecol. Lett., 6, 1-12.

Pacala, S. \& Roughgarden, J. (1982). Spatial heterogeneity and interspecific competition. Theor. Pop. Biol., 21, 92-113.

Tilman, D. (1994). Competition and biodiversity in spatially structured habitats. Ecology (Tempe), 75, 2-16.

Manuscript received 15 October 2002

First decision made 22 November 2002

Manuscript accepted 16 December 2002

\section{APPENDIX. DERIVATION OF THE GROWTH-DENSITY COVARIANCE FORMULA}

We begin with eqn 9 and assume that the invader has reached an equilibrium pattern of relative density. Thus,

$v_{i x}=\sum_{y} k(x-y) \frac{\lambda_{i y}}{\tilde{\lambda}_{i}} v_{i y}$,

where the sum runs over all space.

We assume small perturbations from spatial uniformity in $v_{i x}$ and $\lambda_{i x}$, defining

$u_{i x}=v_{i x}-1$

$\zeta_{i x}=\frac{\lambda_{i x}}{\bar{\lambda}_{i}}-1$

where $u_{i x}$ and $\zeta_{i x}$ are $O(\sigma)$ and $\sigma$ is a small parameter ${ }^{2}$. (By $g(x)=O\left(\sigma^{2}\right)$, we mean that $\left|\frac{g(x)}{\sigma^{2}}\right|$ can be made less than or equal to any positive constant $K$ for $\sigma^{2}$ small enough.) Substituting these definitions into the equation for the invader relative density at equilibrium, eqn 17 , and simplifying, we find

$u_{i x}=\frac{1}{1+\psi} \sum_{y} k(x-y)\left(u_{i y}+\zeta_{i y}+u_{i y} \zeta_{i y}-\psi\right)$,

where

$\psi=\frac{\tilde{\lambda}_{i}-\bar{\lambda}_{i}}{\bar{\lambda}_{i}}=\frac{\operatorname{Cov}\left(\lambda_{i}, v_{i}\right)}{\bar{\lambda}_{i}}$.

The growth-density covariance equals $\bar{\lambda}_{i}$ times $\zeta_{i} u_{i}$, so, apart from the proportionality constant $\bar{\lambda}_{i}$, we could find $\operatorname{Cov}\left(\lambda_{i}, v_{i}\right)$ at lag $d$ by multiplying both sides of eqn 20 by $\zeta_{i, x-d}$ and taking the spatial average. (We will ultimately want only the covariance at lag 0 , but it is helpful to solve for the covariance as a function of lag, as we want to take the Fourier transform.) However, if the system is spatially stationary, the spatial average will be equal to an expectation over an ensemble of replicates. We therefore set lag $d$ equal to $x$, so that we are multiplying by $\zeta_{i 0}$, and take the expectation instead. Denoting the expected value by $\langle\cdot\rangle$, we define

$\phi(x)=\left\langle\zeta_{i 0} u_{i x}\right\rangle$

$R(x)=\left\langle\zeta_{i 0} \zeta_{i x}\right\rangle$

$\eta(x)=\left\langle\zeta_{i 0} u_{i x} \zeta_{i x}\right\rangle$,

and noting that $\left\langle\zeta_{i 0}\left(u_{i y} \zeta_{i j}\right)\right\rangle=\left\langle\zeta_{i 0}\left(u_{i y} \zeta_{i y}-\psi\right)\right\rangle$, we find

$\phi(x)=\frac{1}{1+\psi} \sum_{y} k(x-y)[\phi(y)+R(y)+\eta(y)]$.

This is a convolution and is most easily solved by taking the Fourier transform, which turns convolutions into

\footnotetext{
${ }^{2}$ The formalism presented in Chesson (2000a) assumes that $E$ varies over a finite interval of length $\sigma$.
} 
products. We use the following definition of the discrete Fourier transform:

$\tilde{f}\left(\omega_{s}\right)=\sum_{j=-N / 2+1}^{N / 2} f\left(x_{j}\right) \mathrm{e}^{-\mathrm{i} \omega_{s} x_{j}}$,

$f\left(x_{n}\right)=\frac{1}{N} \sum_{s=-N / 2+1}^{N / 2} \tilde{f}\left(\omega_{s}\right) \mathrm{e}^{\mathrm{i} \omega_{s} x_{n}}$,

where $N$ is the number of lattice points, $\Delta$ is the distance between lattice points, $x_{j}=j \Delta$, and $\omega_{s}=s(2 \pi) /(N \Delta)$. Taking the discrete Fourier transform of eqn 25, we obtain

$\tilde{\phi}\left(\omega_{s}\right)=\frac{1}{1+\psi} \tilde{k}\left(\omega_{s}\right)\left[\tilde{\phi}\left(\omega_{s}\right)+\tilde{R}\left(\omega_{s}\right)+\tilde{\eta}\left(\omega_{s}\right)\right]$

and thus

$\tilde{\phi}\left(\omega_{s}\right)=\frac{\tilde{k}\left(\omega_{s}\right)\left[\tilde{R}\left(\omega_{s}\right)+\tilde{\eta}\left(\omega_{s}\right)\right]}{1-\tilde{k}\left(\omega_{s}\right)+\psi}$.

$\operatorname{Cov}\left(v_{i}, \lambda_{i}\right)$ at lag 0 is simply $\bar{\lambda}_{i} \phi(0)$, so all that remains is to take the inverse Fourier transform of eqn 29 and to evaluate it at $x=0$ :

$\phi(0)=\frac{1}{N} \sum_{s=-N / 2+1}^{N / 2} \frac{\tilde{k}\left(\omega_{s}\right)\left[\tilde{R}\left(\omega_{s}\right)+\tilde{\eta}\left(\omega_{s}\right)\right]}{1-\tilde{k}\left(\omega_{s}\right)+\psi}$.

As we only seek an approximation to $\mathrm{O}\left(\sigma^{2}\right)$, we can drop $\tilde{\eta}(\omega)$ from the numerator and $\psi$ from the denominator in eqn 30. Thus,

$\operatorname{Cov}\left(\lambda_{i}, v_{i}\right)=\frac{\bar{\lambda}_{i}}{N} \sum_{s=-N / 2+1}^{N / 2} \frac{\tilde{k}\left(\omega_{s}\right) \tilde{R}\left(\omega_{s}\right)}{1-\tilde{k}\left(\omega_{s}\right)}$.
We can relate this expression more directly to our rate of increase perturbation, $\zeta_{i x}$, by noting that $R(x)$ is the spatial autocorrelation of $\zeta_{i}$ at lag $x$. This allows us to use the Wiener-Khinchin theorem, which states that the autocorrelation of a function at lag $x$ is equal to the inverse Fourier transform of the function's spectral density, evaluated at $x$. Thus,

$R\left(x_{n}\right)=\frac{1}{N} \sum_{j=-N / 2+1}^{N / 2} S_{\zeta}\left(\omega_{j}\right) \mathrm{e}^{\mathrm{i} \omega_{j} x_{n}}$,

where the spectral density of $\zeta_{i}, S_{\zeta}\left(\omega_{j}\right)$, equals $\frac{1}{N}\left|\tilde{\zeta}_{i}\left(\omega_{j}\right)\right|^{2}$, and

$\tilde{R}\left(\omega_{s}\right)=S_{\zeta}\left(\omega_{s}\right)$.

Thus,

$\operatorname{Cov}\left(\lambda_{i}, v_{i}\right)=\frac{\bar{\lambda}_{i}}{N} \sum_{s=-N / 2+1}^{N / 2} \frac{\tilde{k}\left(\omega_{s}\right) S_{\zeta}\left(\omega_{s}\right)}{1-\tilde{k}\left(\omega_{s}\right)}$.

If $S_{\zeta}\left(\omega_{s}\right)$ and $\tilde{k}\left(\omega_{s}\right)$ do not contain any Kronecker delta functions $\left(\delta_{i j}=1\right.$ if $i=j$, 0 if $\left.i \neq j\right)$, then it is safe to go to the limit of an infinite domain $(N \rightarrow \infty)$ :

$\operatorname{Cov}\left(\lambda_{i}, v_{i}\right)=\bar{\lambda}_{i} \frac{1}{2 \pi} \int_{-\pi}^{\pi} \frac{\tilde{k}(\omega) S_{\zeta}(\omega)}{1-\tilde{k}(\omega)} \mathrm{d} \omega$.

(We have here set $\Delta$ to 1 .) This is our primary result. 